\title{
Visualising Complex Polynomials: A Parabola Is but a Drop in the Ocean of Quadratics
}

\author{
Harry Wiggins ${ }^{1}$, Ansie Harding ${ }^{1} \&$ Johann Engelbrecht ${ }^{2}$ \\ ${ }^{1}$ Department of Mathematics and Applied Mathematics, University of Pretoria, Pretoria, South Africa \\ ${ }^{2}$ Department Science, Mathematics and Technology Education, University of Pretoria, Pretoria, South Africa \\ Correspondence: Johann Engelbrecht, Department of Science, Mathematics and Technology Education, University of \\ Pretoria, 0002 Pretoria, South Africa.
}

Received: August 1, 2018 Accepted: August 21, 2018 Online Published: November 28, 2018

doi:10.5539/jmr.v10n6p91 URL: https://doi.org/10.5539/jmr.v10n6p91

\begin{abstract}
One of the problems encountered when teaching complex numbers arises from an inability to visualise the complex roots, the so-called "imaginary" roots of a polynomial. Being four dimensional, it is problematic to visualize graphs and roots of polynomials with complex coefficients in spite of many attempts through centuries. An innovative way is described to visualize the graphs and roots of functions, by restricting the domain of the complex function to those complex numbers that map onto real values, leading to the concept of three dimensional sibling curves. Using this approach we see that a parabola is but a singular case of a complex quadratic. We see that sibling curves of a complex quadratic lie on a three-dimensional hyperbolic paraboloid. Finally, we show that the restriction to a real range causes no loss of generality.
\end{abstract}

Keywords: complex zeroes, complex polynomials

\section{Introduction}

For centuries mathematicians have spent time and energy on investigating two issues concerning polynomials

- how to visualize complex zeroes

- how to visualize polynomials with complex coefficients.

We address the first of these issues for a range of well-known functions in an elegant way. We show, for example, that the parabola as we know it is part of twin curves in an expanded three dimensional world. We illustrate how the twin curve can be used to visualize complex zeros of the parabola. The second issue is addressed for a quadratic polynomial in such a way that new insights into the shape of a quadratic polynomial are gained. The members of the family of complex quadratics do not all display the typical parabolic shape.

Since Descartes in 1637, in his Discourse, gave a way to link an algebraic formula of a function to a picture of the function, roots of a function have been visualized as the points of intersection with the "floor" (horizontal axis or plane). Almost 200 years later, in 1814, Argand gave geometrical significance to complex numbers by interpreting $i$ as a rotation of the real line through $90^{\circ}$. The plane for representing complex numbers is referred to as the Argand plane.

So we came to have two planes, the Cartesian plane that can be used for showing the graph of a function and where its real roots lie and the Argand plane that gives a visual representation of complex numbers. The problem with these two distinct planes is that if a function has complex roots, we can see these roots on the Argand plane but cannot see where they are with respect to the graph of the function in the Cartesian plane. Since the graph of a complex function on a complex domain is four-dimensional, visualization is not evident.

Finding ways of reading the complex roots of a function from a sketch on the Cartesian plane has been a long-standing and popular topic. Harding and Engelbrecht (2007a) followed the historical journey of finding roots of complex functions, both algebraically and graphically and essentially discussed four ways to visualize the complex roots of polynomial equations, all informative but somewhat artificial.

- Superimposing the Argand plane onto the Cartesian plane (Crawley, 1918; Gleason, 1910; Gehman, 1941; Long, 1972; Ward, 1937; Yanosik, 1936).

- Placing the two planes next to each other and tracing the complex roots by moving from the one plane to the other (Ward, 1937). 
- Taking the modulus of the function values (a real number) to get rid of the fourth dimension and looking at the 3-dimensional surface (Long, 1971; Long, 1972; Long \& Hern, 1989).

- Colour coding of complex numbers to give a visual representation of a function mapping complex numbers onto complex values (Velleman, 2017).

Although these representations are visual and illustrative, the approaches simply offer a compromise for not being able to fully visualize graphs of functions that map complex numbers onto complex numbers. The question of "what cuts where?" remains unsatisfactorily answered.

Seemingly almost unobserved, a new idea appeared in print in 1951, in an American secondary school textbook (H. Fehr, 1951). This approach was further developed in Harding and Engelbrecht (2007b) which led to the idea of sibling curves, which turned out to be a rich and useful way of visualizing zeroes of polynomials and other well-known functions as well as visualizing complex functions in three dimensions.

A polynomial $f$ that maps complex numbers onto complex numbers has $n$ complex roots. This is a result that follows from the Fundamental Theorem of Algebra. For example, $f(z)=z^{2}+1$, has two imaginary roots $i$ and $-i$. Two important observations need to be emphasized:

- If we speak about complex roots of a polynomial, we implicitly include complex numbers in the domain of the function.

- Furthermore, almost always have the function values of the polynomial been considered as real numbers.

These observations motivate the following approach: If we restrict the domain of $f$ to those complex numbers that map onto real values, the function can be represented in three dimensions on this restricted domain with the domain in the horizontal plane (the complex plane) and the range along the vertical axis.

In particular, a polynomial $f$ can be written in the form $w=f(z)=f(x+i y)=g(x, y)+i h(x, y)$ for some polynomials $g$ and $h$. If $f$ maps the complex number $x+i y$ onto a real number then $h(x, y)=0$. If we restrict the domain of the function $f$ to all points in the $x y$-plane such that $h(x, y)=0$, the condition $h(x, y)=0$ defines a curve(s) in the Argand plane (the horizontal plane). The function $f$ with these curves as domain form the sibling curves. The question of how to visually represent the zeroes of the function is answered by means of the sibling curves. An example function illustrates the concept.

If $f(z)=z^{2}+2 z+2$ and $z$ is a complex number of the form $x+i y$ then

$$
f(z)=\left(x^{2}-y^{2}+2 x+2\right)+2 i y(x+1)
$$

So $f(z)$ is real in the plane $y=0$ (the Cartesian plane) and in the plane $x=-1$ (a plane perpendicular to the Cartesian plane). In the real plane $y=0$ the function values are given by

$$
f(x)=x^{2}+2 x+2, x \in \mathbb{R},
$$

which is the well-known parabola that we have always considered as the entire graph.

However, in the plane $x=-1$, perpendicular to the Cartesian plane, the function is $f(y)=-y^{2}+1, y \in \mathbb{R}$. It is then clear that the parabola as we know it is part of Siamese twin curves, defined in planes perpendicular to each other. These twin curves are called the sibling curves of the function. The function has complex roots $z=-1 \pm i$, and visually it is evident that these roots are situated where the function $f(y)$ cuts the complex (horizontal) plane. Both curves have the typical parabolic shape.

The ideas of Fehr (1951), exposed here, were picked up more than thirty years later by Vest (1985) reiterated for the general case of $f(z)=A z^{2}+B z+C$, but have remained dormant since, to the best of our knowledge. In a more recent article by Ballew (2018), eighteen ways to solve quadratic equations by analytic and graphic methods were discussed, sub-headed by "Including those you may never have seen". It was noticeable that although there is a passing reference to Fehr's book, this approach is absent. Another recent discussion in the online Teacher2Teacher (2003) forum speculates about the existence of what the enquirer refers to as phantom curves, which seem to be what we call sibling curves, but no definite conclusion was reached as to their existence.

Expanding on the idea outlined above, Harding and Engelbrecht (2007b) developed sibling curves of a number of wellknown functions, including cubics, quartics, exponential, trigonometric and hyperbolic functions. Interestingly, the sibling curves of a sine or cosine function are hyperbolic functions and the siblings of a circle are hyperbolas. 


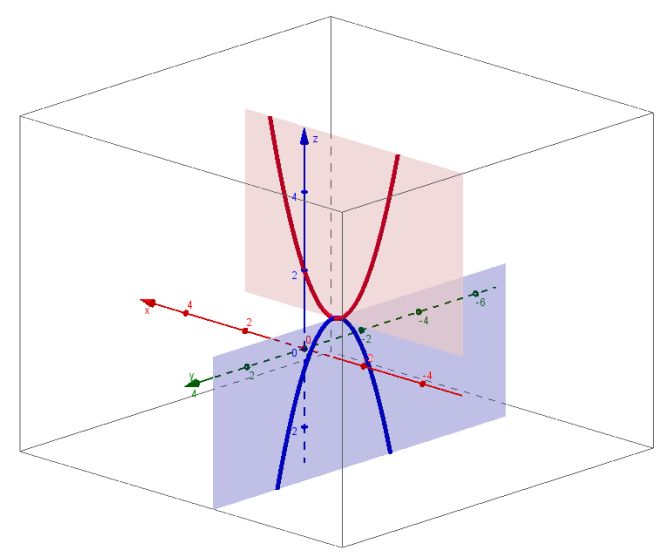

Figure 1. The quadratic function $f(z)=z^{2}+2 z+2$

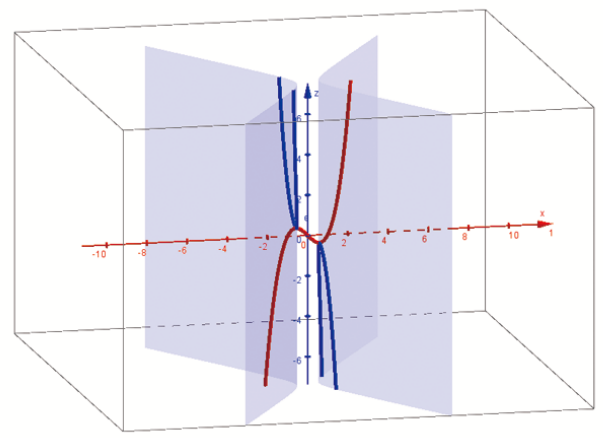

Figure 2. Sibling curves of $f(z)=z^{3}-z$.

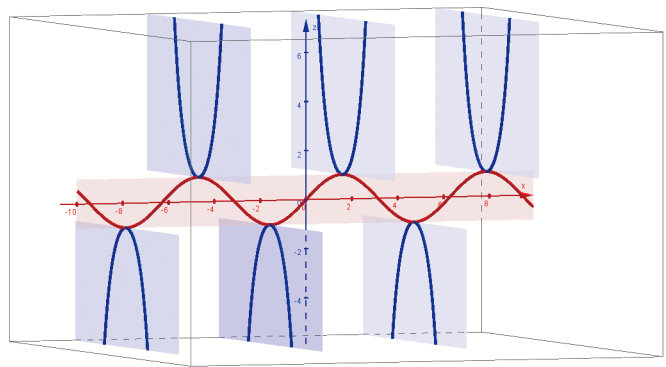

Figure 3. Sibling curves of $f(z)=\sin z$.

Looking at the sibling curves of $f(z)=z^{n}-1$, one gets an enlightening new picture of the $n^{\text {th }}$ roots of 1 . In Figure 5, we visualize the $6^{\text {th }}$ roots of 1 , as the positions where the sibling curves cut the complex plane.

The work on sibling curves was given a more solid mathematical founding in (Wiggins, Harding \& Engelbrecht, 2015) in which the main result proved is that a polynomial with degree $\mathrm{n}$ (real or complex coefficients) has exactly $n$ sibling curves.

The mathematical development for quadratics was expanded in (Wiggins, Harding \& Engelbrecht, 2017) where it was proved that siblings of quadratics with real coefficients are congruent parabolas, touching at the turning point.

\section{Complex Quadratics}

In the case of quadratic polynomials with complex coefficients it was proved by Wiggins, Harding \& Engebrecht (2017) that the quadratic $f(z)=a z^{2}+b z+c, a, b, c \in \mathbb{C}$ and $a \neq 0$ has two siblings that intersect if and only if $4|a|^{2} c-b^{2} \bar{c}$ is a 


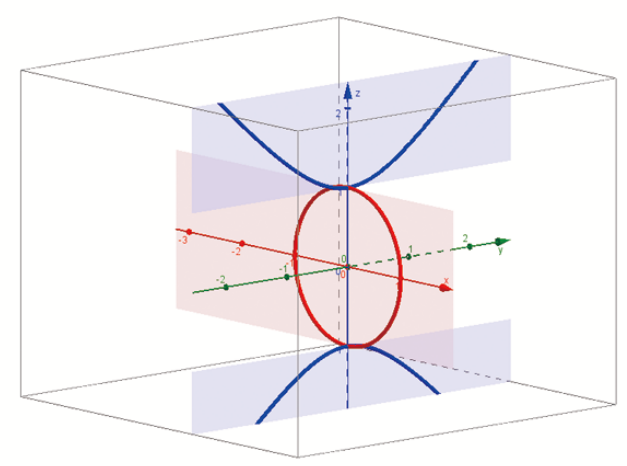

Figure 4. Sibling curves of a circle.

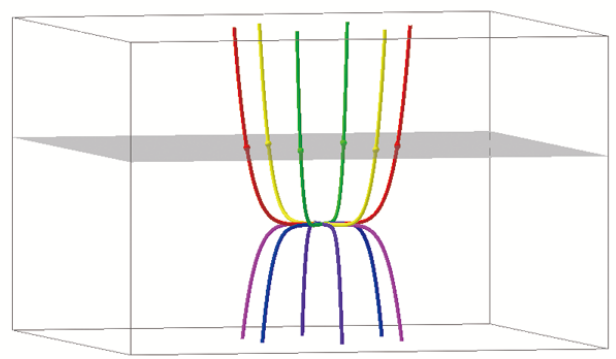

Figure 5. Sibling curves of the function $f(z)=z^{6}-1$.

real number and that the projections on the domain are hyperbolic. An example illustrates the hyperbolic projections.

Consider $f(z)=(z-1)(z-i)=z^{2}-(1+i) z+i$. Let $z=x+i y$, then

$$
f(z)=(x+i y)^{2}-(1+i)(x+i y)+i=\left(x^{2}-y^{2}-x+y\right)+i(2 x y-x-y+1) .
$$

If $f(z)$ is real then $2 x y-x-y+1=0$ implying that $y=\frac{x-1}{2 x-1}$ which means that the projection is hyperbolic. See Figure 6 .

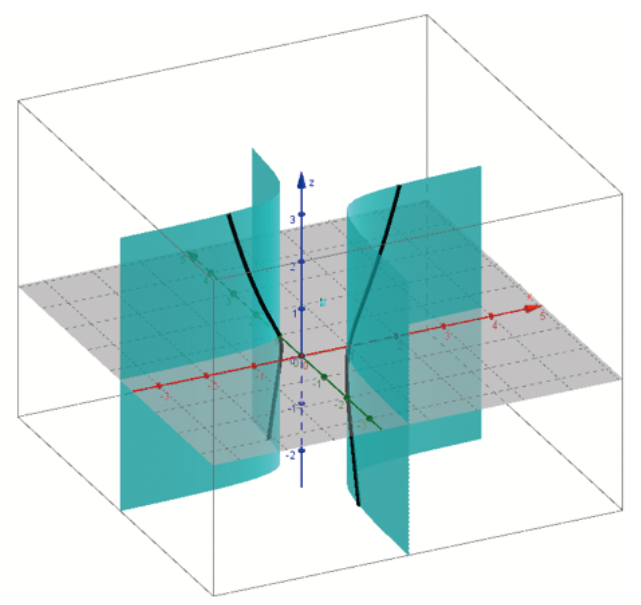

Figure 6. Sibling curves of $f(z)=(z-1)(z-i)$.

It is clear from Figure 6 that the siblings cut the floor at 1 and $i$. 
In this example we notice two important aspects in which these curves differ from quadratics with real coefficients:

- The sibling curves of these quadratics are no longer parabolas but they are still congruent (proved by Wiggins, Harding \& Engelbrecht, 2017).

- The projection of these sibling curves on the complex domain is a hyperbola (Figure 6) and not two perpendicular straight lines as was the case with real quadratic polynomials.

The projection of a quadratic with real coefficients as two perpendicular lines proves to be the degenerate case of the hyperbola. For higher degree polynomials the siblings are not always congruent e.g. the cubic polynomial $f(z)=z^{3}-z$ has three siblings that are not congruent (Figure 2).

\section{Hyperbolic Paraboloid}

In summary, we notice that for quadratic polynomials two things can happen. One scenario is that the sibling curves meet. In this case they are two planar parabolas, each sibling curve lying in its own plane. The second scenario is that the sibling curves never meet and here we have two curves that are not parabolas - the projection on the domain is hyperbolic. The two sibling curves are congruent in both cases.

By using a translation, scaling or rotation on the function, we need, without any loss of generality, only consider the quadratic polynomial $f(z)=z^{2}+c$ for some complex number $c$. If $z=x+i y$, then $f(z)=(x+i y)^{2}+c=x^{2}-y^{2}+$ $\operatorname{Re}(c)+i(2 x y+\operatorname{Im}(c))$. Hence the sibling curves always lie on a scaling, translation or rotation of the hyperbolic paraboloid $z=x^{2}-y^{2}$ (saddle shaped surface or "Pringle").

In Figure 7 we see the typical scenario when the two sibling curves meet. We know that in this case the curves are two congruent parabolas that meet in a point. Notice that the two parabolas meet in the saddle point of the hyperbolic paraboloid - both parabolas' turning point.

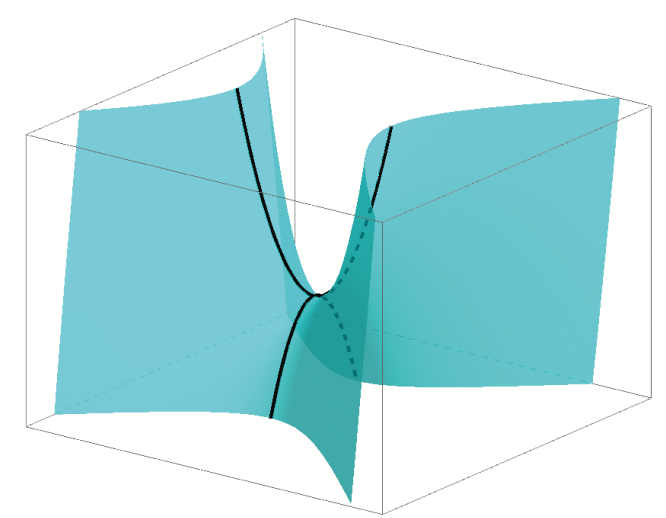

Figure 7. Sibling curves of $f(z)=z^{2}-1$ on a hyperbolic paraboloid.

In Figure 8 we see the typical scenario when the two sibling curves do not meet (except in one case). We know that in this case we do not get two parabolas. However, the sibling curves are still congruent and they lie on the surface of a hyperbolic paraboloid.

An animation of the sibling curves of the quadratic $f(z)=z^{2}+2 z+(1+i k)$ can be seen at https://cardanogroup.wordpress.com/. In Figure 8 we sketched five snapshots of the sibling curves of this quadratic for various real values of $k$. By varying $k$ from -2 to 2 and then in reverse, we see the evolution of sibling curves on a saddle surface, the hyperbolic paraboloid. Notice that when $k=0$ the two sibling curves are parabolas that intersect in a point. This is the only value of $k$ for which this happens.

Quite surprisingly we notice from this animation (or looking at the snapshots of the animation) that one half of the parabola joins up with another half of the other parabola to form new sibling curves when $k \neq 0$.

\section{General Case}

In this paper we considered a three-dimensional cut of the four-dimensional graph by only considering those complex numbers in the domain of which the function values are real. This is a special case in that we do not see the complete four-dimensional object. Harding and Engelbrecht (2007c) also explored the other way of cutting through the graph by considering only those points in the domain where the function values are purely imaginary, giving more clarity on the 


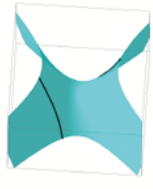

(a) $k=-2$

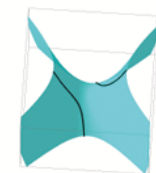

(b) $k=-1$

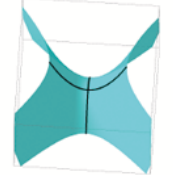

(c) $k=0$

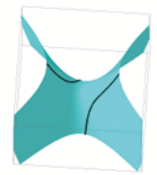

(d) $k=1$

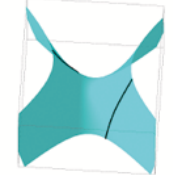

(e) $k=2$

Figure 8. Snapshots of some sibling curves of $f(z)=z^{2}+2 z+(1+i k)$.

graph itself. However, by considering polynomials with complex coefficients, as in this paper, this exercise now seems redundant.

If we let

$$
f(z)=a z^{2}+b z+c=k e^{i \theta} \in \mathbb{C}
$$

then

$$
a z^{2} e^{-i \theta}+b z e^{-i \theta}+c e^{-i \theta}=k \in \mathbb{R}
$$

which is exactly the case that was covered in this paper.

This fresh insight implies that by only considering the case where the function values are real we place no limitation on the images created representing all quadratics - nothing new will be seen if we include complex function values in the range instead of being limited to real function values.

\section{Conclusion}

The approach outlined here gives a fresh view to graphical representation of complex roots of functions and a general understanding of the functions that we deal with every day. It expands the two-dimensional world of the well-known functions perceived thus far and, once aware of the existence of the so-called sibling curves, it seems to be somewhat restrictive to view functions in their previously perceived solitary way. The interpretation of complex roots as opened up by the introduction of sibling curves, unlike other attempts discussed in the first part of the paper, seems to be the natural way of visually representing roots. The approach assists especially for contextualising the Fundamental Theorem of Algebra and visualizing the positioning of complex roots with respect to the function and its other siblings. The value of this approach is also evident from the realization that by considering the special case where the function values are real there is no loss of generality and it does not limit our view of the complete graph.

New insights were gained for the quadratic with complex coefficients. Either the two sibling curves meet in which case they are two planar parabolas - with each sibling curve lying in its own plane - or the sibling curves never meet and the two curves are not parabolas. The projection of the sibling curves on the domain is hyperbolic and the two sibling curves are congruent in both cases.

Finally, we obtain a new perspective on the shape of a parabola as traditionally acknowledged. From the work presented here (Figure 8 in particular) it appears that a parabola consists of two halves, each the degenerate case of part of a sibling curve. We are left with the question as to how this interpretation fits in with the traditional locus definition of a parabola.

\section{References}

Ballew, P. (2018, July 26). Solving quadratic equations by analytic and graphic methods; including several methods you may never have seen. Retrieved from http://www.pballew.net/quadsol.doc

Crawley, E. S. (1918). Relating to the graph of a cubic equation having complex roots. Amer. Math. Monthly, 25(6), 268-269. https://doi.org/10.2307/2972885

Fehr, H. (1951). Secondary Mathematics. Heath, Washington, DC.

Gleason, G. E. (1910). A simple method for graphically obtaining the complex roots of a cubic equation. Ann. Math. 2nd series, 3, 95-96. https://doi.org/10.2307/1967322

Gehman, H. M. (1941). Complex roots of a polynomial equation. Amer. Math. Monthly, 4, 237-239. 
https://doi.org/10.1080/00029890.1941.11991104

Harding, A., \& Engelbrecht, J. (2007a). Sibling curves and complex roots 1: Looking back. International Journal of Mathematical Education in Science and Technology, 38(7), 963-973.

Harding, A., \& Engelbrecht, J. (2007b). Sibling curves and complex roots 2: Looking ahead. International Journal of Mathematical Education in Science and Technology, 38(7), 975-985.

Harding, A., \& Engelbrecht, J. (2007c). Sibling curves 3: Imaginary siblings and tracing complex roots. International Journal of Mathematical Education in Science and Technology, 40(7), 975-985.

Long, C. A. (1971). A note on the geometry of zeros of polynomials. Math. Mag, 3, 157-159. https://doi.org/10.1080/0025570X.1971.11976129

Long, C. A. (1972). The quadratic polynomial and its zeros. The Two-Year College Math. Journal, 3(1), $23-29$. https://doi.org/10.2307/3026793

Long, C., \& Hern, T. (1989). Graphing the complex zeros of polynomials using modulus surfaces. 20(2), 98-105.

Teacher2Teacher. (2003). The geometry of the complex roots of graphs. Retrieved from http://mathforum.org/t2t/message. taco?thread=12605message $=1$

Ward, J. A. (1937). Graphical representation of complex roots. Nat. Math. Mag, 11(7), 297-303. https://doi.org/10.2307/3028785

Velleman, D. J. (2017). The Fundamental Theorem of Algebra: A visual approach. Retrieved from http://www.cs.amherst.edu/ djv/FTAp.pdf

Vest, F. (1985). Graphing the complex roots of a quadratic equation. The College Mathematics Journal, 16(4), $257-261$. https://doi.org/10.1080/07468342.1985.11972891

Wiggins, H., Harding, A., \& Engelbrecht. (2017). Sibling curves of quadratic polynomials. Quaest. Math, 40(2), 215-223. https://doi.org/10.2989/16073606.2017.1284910

Wiggins, H., Harding, A., \& Engelbrecht, (2015). Sibling curves of polynomials. Quaest. Math, 38(6), 759-769. https://doi.org/10.2989/16073606.2014.981727

Yanosik, G. A. (1936). A graphical solution for the complex roots of a cubic. Nat. Math. Math, 10(4), 139-140. https://doi.org/10.2307/3028844

\section{Copyrights}

Copyright for this article is retained by the author(s), with first publication rights granted to the journal.

This is an open-access article distributed under the terms and conditions of the Creative Commons Attribution license (http://creativecommons.org/licenses/by/4.0/). 